ÉGYPTE monde arabe

\section{Égypte/Monde arabe}

$2 \mid 1990$

Médiateur et métaphores 1

\title{
Médiateurs et métaphores 1: Introduction
}

\section{(2) OpenEdition}

\section{Journals}

Édition électronique

URL : https://journals.openedition.org/ema/209

DOI : $10.4000 /$ ema.209

ISSN : 2090-7273

\section{Éditeur}

CEDEJ - Centre d'études et de documentation économiques juridiques et sociales

\section{Édition imprimée}

Date de publication : 30 juin 1990

Pagination : 7-10

ISSN : 1110-5097

\section{Référence électronique}

«Médiateurs et métaphores 1: Introduction », Égypte/Monde arabe [En ligne], 2 | 1990, mis en ligne le 08 juillet 2008, consulté le 07 juillet 2022. URL : http://journals.openedition.org/ema/209 ; DOI : https://doi.org/10.4000/ema.209

Ce document a été généré automatiquement le 7 juillet 2022.

Tous droits réservés 


\section{Médiateurs et métaphores 1: Introduction}

1 Le séminaire hebdomadaire de recherche "Connaissance de l'Égypte » proposé par le CEDEJ a porté, durant l'année universitaire 1989-90, sur le thème "Médiateurs et métaphores ", que les organisateurs de ce séminaire, Iman Farag et Alain Roussillon, ont articulé autour d'hypothèses de travail définies, selon leurs propres termes, comme suit :

$2-D u$ point de vue des systèmes de relations entre l'État ou ce qui en tient lieu et se désigne habituellement comme la "société civile ", la notion de "médiateurs» fait sens dans le cadre d'une cohabitation ou d'une compétition entre systèmes d'action dont les protagonistes et les enjeux doivent, dès lors, être identifiés : partis politiques, associations professionnelles, groupes confessionnels ou communautaires qui gèrent différemment leur rapport à l'État et leurs modes d'insertion dans les structures sociales. La logique du déploiement de ces systèmes d'action est susceptible de se traduire par un refus pur et simple du politique, la revendication d'une participation, elle-même " politique » ou non, la constitution d'espaces publics « non politiques » ou encore la restructuration des espaces publics/privés. Il s'agit ici d'engager une réflexion sur les processus de diversification des comportements sociaux de participation, tendant à identifier:

- des espaces de participation et d'interaction en relation avec les lieux formellement ou informellement constitués du pouvoir : législatif, exécutif, judiciaire, mais aussi shilal, "centres de forces ", réseaux souterrains de l'autorité.

- la façon dont se structurent, se combinent ou s'opposent des modes formels ou informels de participation et, en particulier les processus de « formalisation » ou « d'informalisation » des comportements de participation, en prenant en compte, notamment, la dimension régionale ou locale des structures au travers desquelles celle-ci se joue.

- la façon dont peuvent s'articuler les intérêts, individuels ou collectifs, qui s'expriment aux différents niveaux - économique, religieux, politique... - de la pratique sociale, dont il s'agit de comprendre comment ils se projettent dans des systèmes d'action à dominante religieuse, revendicative ou explicitement politique.

$3-$ Au sein des groupes considérés ou du point de vue du fonctionnement des systèmes d'action, la notion de "médiateurs» ou de "médiation» renvoie à la structure de l'autorité et de la 
légitimité qui assure leur cohésion et la pérennité de «l'être-ensemble » qui les caractérise. Selon que l'on envisage des mouvements à dominante "culturelle", des associations professionnelles ou des regroupements à dominance communautaire, on aura affaire à différentes figures de la médiation et du médiateur, susceptibles de s'appuyer, pour l'accomplissement de leur rôle au sein du groupe, sur différents registres allant de la défense de l'identité à la fidélité idéologique ou doctrinale en passant par la défense pure et simple des intérêts matériels des membres du groupe. Dans la mesure où le propre des médiateurs est de pouvoir se situer alternativement dans un " mode » ou dans l'autre, il importe de tenter de les saisir en termes de mouvement ou de processus plutôt que de fonction ou de statut, c'est-à-dire de tenter de concevoir leurs rôles autrement qu'en termes de "porte-parole», "représentants» ou autres "intellectuels organiques ». Il s'agit ici de prendre en compte tout à la fois :

- l'origine - charismatique, familiale, liée à des compétences particulières, déléguée... - de cette légitimité, et le système de relations qu'elle institue entre le " médiateur » dépositaire et gestionnaire des intérêts du groupe et ses membres.

- les modalités symboliques et pratiques de l'exercice de l'autorité au sein du groupe, et en particulier la façon dont les enjeux internes et externes de celui-ci peuvent être articulés.

- les types de mobilité sociale que l'appartenance au groupe rend possible, individuellement ou collectivement, et les divisions du travail au travers lesquelles elle se joue.

- Si l'on s'accorde, avec Pierre Bourdieu, pour désigner la métaphore comme une structure de sens simultanément structurée et structurante, c'est-à-dire d'une part, comme une expression spécifique de la dimension symbolique des comportements et, d'autre part, comme une réalisation également spécifique de la dimension cognitive et interprétative de toute conduite sociale, l'analyse du statut de la métaphore envisagée en relation avec les systèmes d'action conduit à s'interroger sur :

- la façon dont s'opère la mutation des productions individuelles, reprises comme symboles collectifs à travers un processus de fixation et d'interprétation qui rend la métaphore, au sens propre, parlante.

La métaphore doit ici être envisagée comme le système producteur de l'univers de sa propre légitimité, et non pas seulement comme la simple reproduction d'un « déjà là ».

- le système de référents - religieux, «scientifiques »... - à partir duquel s'opère le codage/décodage qui détermine son efficacité et qui permet d'être certain « qu'on pane bien de la même chose ».

- la façon dont elle rend possible, par sa " transitivité " propre et au travers de reformulations successives qui définissent sa " positivité », l'articulation des divers niveaux d'intérêts mobilisés par les systèmes d'action collectifs.

Dans cette perspective, l'on peut risquer une double proposition permettant d'articuler les deux notions :

- la métaphore serait le mode privilégié par lequel les intérêts spécifiques qui s'expriment dans des systèmes d'action visant à une participation au politique non reconnue comme telle, s'énoncent en se niant comme intérêts et en s'affirmant comme valeur absolue.

- la métaphore serait le mode privilégié au travers duquel peut s'exercer la médiation dans la mesure où c'est sa capacité à en gérer les enjeux qui légitime le médiateur dans sa fonction de truchement entre le groupe et le monde extérieur.

6 A l'articulation de ces deux propositions, la métaphore pourrait être conçue comme une expression allégorique de la connaissance/représentation des lieux de pouvoir, autorisant à en traiter comme une " manière de faire " parmi d'autres.

7 Quelques-unes des communications présentées au cours de ce séminaire ont été rassemblées dans cette seconde livraison d'Égypte/Monde arabe et feront également 
l'objet du numéro à venir. Certaines ont été élaborées à partir de la plate-forme définie plus haut; d'autres représentent des étapes de travaux en cours et ne se réclament pas de la même problématique.

Dans la présente livraison, Iman Farag s'interroge sur le rôle de médiation des prédicateurs, médiation envisagée non seulement dans le contexte du rituel qu'ils «mettent en scène » mais aussi du point de .vue de leur ancrage social. Relativisant l'impact du dirigisme étatique à l'égard des prédicateurs, l'auteur propose un cadre d'analyse qui tend à dépasser ce que pourrait avoir de réductrice une lecture politicoreligieuse du prône, du rôle des prédicateurs et de leurs pratiques.

Gamal Abd al-Gawad rappelle les étapes de l'islamisation du Soudan en insistant sur le rôle joué par les confréries dans ce processus : leur réaction aux menaces que faisait peser sur leur existence l'essor du mouvement mahdiste, lequel se présentait à la fois comme alternative à ces communautés et seule expression authentique de l'Islam, a favorisé l'émergence d'un islam communautariste qui allait se répercuter par la suite sur la formation des partis politiques soudanais.

Pierre-Jean Luizard dresse un panorama exhaustif du soufisme égyptien contemporain, qu'il définit comme le " principal vecteur » - notamment à travers les mawlid - « de la piété populaire ». Il retrace étape après étape comment, par le biais d'une législation toujours plus contraignante, l'État a cherché, dès l'époque ottomane, à domestiquer cette expression de la «mystique organisée ». Il s'interroge pour finir sur les défis que pose la modernité aux confréries soufies : faut-il, à leur égard, parler de déclin ou de renaissance?

11 La contribution de Kenneth Brown, anthropologue enseignant à l'université de Manchester et spécialiste du monde arabe, paraît se situer à première vue hors du thème. En réalité, il s'y rattache on ne peut plus clairement. Son personnage central, Ameur le Tunisien, est l'intercesseur par excellence, le médiateur entre sa société et l'étranger, le truchement sans lequel le chercheur venu d'un autre horizon ne pourrait avoir accès à la communauté qu'il observe, analyse, explique. Ameur, c'est donc le joint, « informateur » et " compagnon » à la fois, sans qu'il y ait de sa part «trahison » vis-àvis de sa propre société ou duperie par rapport à celui qu'il renseigne. Ce n'est pas un « agent double » mais un témoin qui fait partager son témoignage.

Le $\mathrm{n}^{\circ} 3$ d'Égypte/Monde arabe, à paraître début 1991, rassemblera sur le même thème les contributions de Jean-Charles Depaule («Le vêtement comme métaphore?»), d'Anne Kazazian («Les logiques associatives dans la communauté arménienne orthodoxe d'Égypte »), de Nasr Abu Zayd (" Mécanismes et fondements intellectuels du discours religieux contemporain ») et d'Alexandre Popovic (sur les relations entre le pouvoir yougoslave et la communauté musulmane de Yougoslavie entre 1945 et 1989.) 